\title{
Fundamental Symmetry of Barium Titanate Single Crystal Determined Using Energy-Filtered Scanning Convergent Beam Electron Diffraction
}

\author{
Yu-Tsun Shao ${ }^{1}$ and Jian-Min Zuo ${ }^{1,2}$ \\ 1. Department of Materials Science and Engineering, University of Illinois, Urbana, Illinois, USA \\ 2. Frederick Seitz Materials Research Laboratory, University of Illinois, Urbana, Illinois, USA
}

Structural phase transitions in ferroelectric perovskites have been intensely investigated over many decades owing to their large impact on physical properties as well as technology applications. Barium titanate $\left(\mathrm{BaTiO}_{3}\right)$ is a ferroelectric, and considered as a classic example of displacive phase transition. Atomic displacements accompanied by polarization change, $\mathrm{BaTiO}_{3}$ successively transforms from a high-temperature paraelectric cubic phase to three low-temperature ferroelectric phases with tetragonal $(\mathrm{T})$, orthorhombic $(\mathrm{O})$, and rhombohedral (R) symmetries. However, various experimental and theoretical studies suggested order-disorder character $[1,2]$, in which the atomic positions change by performing thermally activated jumps between two or more equilibrium positions. Thus, there is a need to probe the crystal symmetry in nanoscale or even at unit-cell level in order to understand the physics of phase transition.

To map out symmetry variations across region of interest in nanoscale, we used energy-filtered scanning convergent beam electron diffraction (EF-SCBED) along with a new proposed algorithm for symmetry quantification. EF-SCBED is based on automated recording of energy-filtered CBED patterns on the CCD camera while scanning over the user-defined region with a nanometer-sized focused electron beam. Each CBED pattern was energy-filtered using an in-column Omega Filter with an energy window of $10 \mathrm{eV}$ to reduce inelastic scattering background. The symmetry quantification is based on the method proposed by Kim \& Zuo [3], using the normalized cross-correlation coefficient $(\gamma)$ of intensities between a pair of CBED discs. However, it is possible to have different $\gamma_{g}$ values for different pair of reflections in an experimental CBED pattern. To provide one $\gamma$ for each CBED pattern, our new algorithm weights each $\gamma_{g}$ value with respect to the disc intensities.

For example, we take Si [110] CBED pattern as a test. Figure 1(a) shows the symmetry variation map of a scanned area of $25 \mathrm{~nm} \times 25 \mathrm{~nm}$. Take the CBED pattern of highest $\gamma$ value as an example (corresponds to white region in figure 1(a)), figure 1(b) shows the image processing procedure for mirror symmetry quantification. The symmetry related pairs of diffraction discs from (A, A') to $\left(G, G^{\prime}\right)$ are selected (orange circles) about the mirror plane (blue circles). The table in figure 1(b) list out each $\gamma_{g}$ value and the corresponding summed intensities. Applying the equation $\gamma_{\text {representative }}=\frac{\sum_{g} I_{g} \gamma_{g}}{\sum_{g} I_{g}}$, the weighted cross-correlation value of this pattern is $\gamma=98.1 \%$. The above method was applied to the study of symmetries of $\mathrm{BaTiO}_{3}$ single crystal, where the spontaneous polarization $\left(\mathrm{P}_{\mathrm{s}}\right)$ lies in the mirror plane.

We identified regions of highest symmetry from a total of 625 CBED patterns over area of $25 \mathrm{~nm} x$ $25 \mathrm{~nm}$. Figure 2 is a collection of most symmetrical CBED patterns of $\mathrm{BaTiO}_{3}$ taken along pseudocubic incidence $[100]_{\mathrm{pc}}$ at different temperatures. In the $\mathrm{T}(P 4 m m), \mathrm{O}(A m m 2)$, and $\mathrm{R}(R 3 m)$ phases, $\mathrm{P}_{\mathrm{s}}$ lies in one of the $\langle 100\rangle_{\mathrm{T}},\langle 110\rangle_{\mathrm{O}}$, and $\langle 111\rangle_{\mathrm{R}}$ directions, respectively. Thus for CBED patterns taken along the $[100]_{\mathrm{pc}}$ incidence, we should expect mirror planes normal to $[010]_{\mathrm{T}},[001]_{\mathrm{T}},[011]_{\mathrm{O}},[0 \overline{1} 1]_{\mathrm{O}}$, $[011]_{\mathrm{R}}$, and $[0 \overline{1} 1]_{\mathrm{R}}$ directions.

Figure 2(a) shows the CBED pattern of $\mathrm{T}$ phase taken at room temperature. The mirror plane $\mathrm{m} / /[001]_{\mathrm{T}}$ and $\gamma=98.5 \%$ is consistent with the space group $P 4 \mathrm{~mm}$. Figure 2(b) and 2(c) shows the 
CBED pattern taken approximately at $263 \mathrm{~K}$ (O phase) and $95 \mathrm{~K}$ (R phase) with mirror plane $m / /[011]_{\mathrm{O}}$ and $\gamma=95.8 \%, m / /[011]_{\mathrm{R}}$ and $\gamma=94.6 \%$, respectively. The observed mirror planes are consistent with the space group Amm2 in the $\mathrm{O}$ phase and $\mathrm{R} 3 \mathrm{~m}$ in the $\mathrm{R}$ phase. These imply the existence of true tetragonal symmetry at room temperature, orthorhombic symmetry at $263 \mathrm{~K}$, and rhombohedral symmetry at $95 \mathrm{~K}$. However, we also detected regions with broken symmetries, which was also reported by Tsuda et al. using a similar technique (STEM-CBED method) [4, 5]. In summary, EF-SCBED allows detection of the highest symmetry in a TEM sample and elimination of sample defects and strain. Mirror symmetry is detected with a $1 \mathrm{~nm}$ probe, consistent with the $\mathrm{T}, \mathrm{O}$, and $\mathrm{R}$ symmetry of $\mathrm{BaTiO}_{3}$.

\section{References:}

[1] G. Völkel and K. A. Müller, Phys. Rev. B 76 (2007), 094105.

[2] B. Zalar, V. V. Laguta, and R. Blinc, Phys. Rev. Lett. 90 (2003), 037601.

[3] K. Kim and J. M. Zuo, Ultramicroscopy 124 (2013), 71.

[4] K. Tsuda, R. Sano, and M. Tanaka, Phys. Rev. B 86 (2012), 214106.

[5] K. Tsuda, A. Yasuhara, and M. Tanaka, Appl. Phys. Lett. 103 (2013), 082908.

[6] This work is supported by U.S. Department of Energy under contract DEFG02-01ER45923.
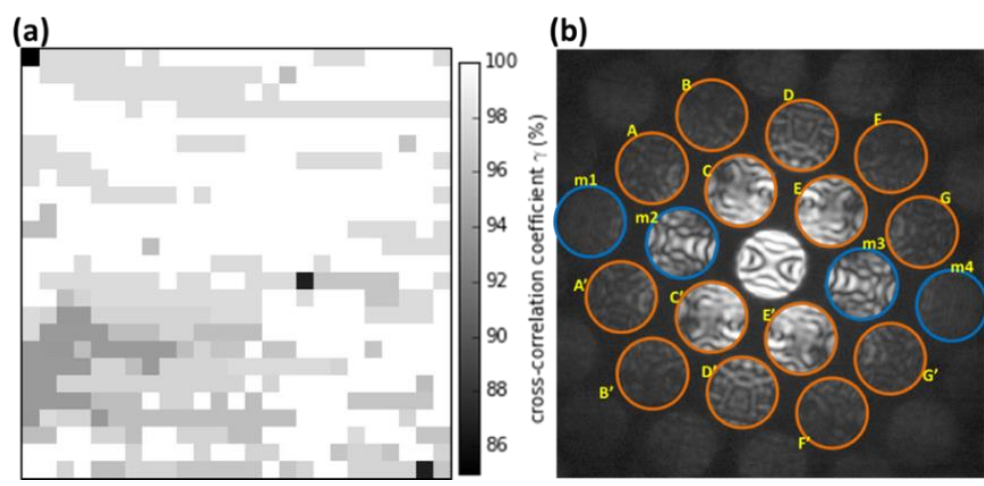

\begin{tabular}{|c|c|c|}
\hline & $Y$ & Sum intensity \\
\hline$\left(A, A^{\prime}\right)$ & 0.961096 & 206236 \\
\hline$\left(B, B^{\prime}\right)$ & 0.925561 & 150530 \\
\hline$\left(C, C^{\prime}\right)$ & 0.993897 & $1.45153 e+006$ \\
\hline$\left(D, D^{\prime}\right)$ & 0.968451 & 393573 \\
\hline$\left(E, E^{\prime}\right)$ & 0.992349 & $1.42561 e+006$ \\
\hline$\left(F, F^{\prime}\right)$ & 0.926832 & 153510 \\
\hline$\left(G, G^{\prime}\right)$ & 0.966375 & 194898 \\
\hline$m 1$ & 0.920665 & 147888 \\
\hline$m 2$ & 0.988652 & 952739 \\
\hline$m 3$ & 0.989302 & 978776 \\
\hline$m 4$ & 0.914988 & 148226 \\
\hline 25
\end{tabular}

Figure 1. (a) Symmetry variation map of the scanned area of $25 \mathrm{~nm} \times 25 \mathrm{~nm}$. (b) Procedure used for quantifying mirror symmetry of a CBED pattern with highest $\gamma$ values (corresponds to white regions in (a)). Pairs of diffraction discs related by mirror are selected and marked by the orange circles from (A, $\left.A^{\prime}\right)$ to $\left(\mathrm{G}, \mathrm{G}^{\prime}\right)$. The diffraction discs on the mirror plane are marked by blue circles from $\mathrm{m} 1$ to $\mathrm{m} 4$. The table list out the $\gamma$ values and the corresponding summed intensities.

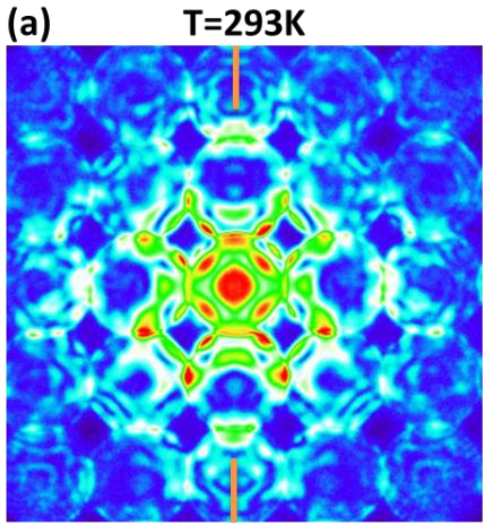

$\mathrm{m} / /[001]_{\mathrm{T}}$

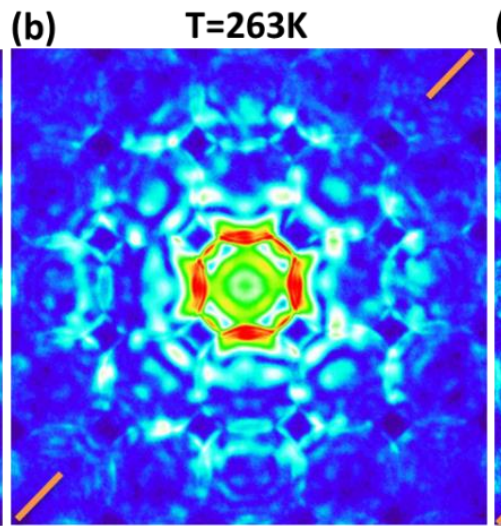

$\mathrm{m} / /[011]_{0}$

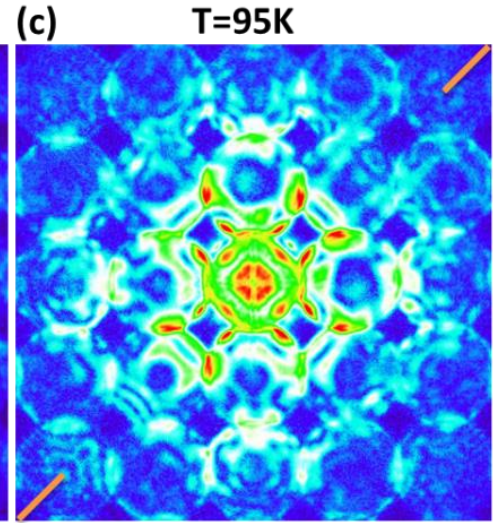

$\mathrm{m} / /[011]_{\mathrm{R}}$

Figure 2. Representative $\mathrm{CBED}$ patterns of $\mathrm{BaTiO}_{3}$ single crystal along [100] $]_{\mathrm{pc}}$ zone axis taken at (a) room temperature ( $\mathrm{T}$ phase) (b) $263 \mathrm{~K}$ (O phase) and (c) $95 \mathrm{~K}$ ( $\mathrm{R}$ phase). The mirror planes are marked by orange lines. 